\title{
Effects of Baduanjin on Essential Hypertension: A Study Protocol for a Randomized Controlled Trial
}

\section{Yuanyuan Guan ( $\nabla$ gyy19900507@163.com )}

Tianjin University of Traditional Chinese Medicine

\section{Yang Hao}

Tianjin First Centeral Hospital

\section{Yun Guan}

Australian National University

\section{Dongjun Wang}

Tianjin University of Traditional Chinese Medicine

\section{Xuan Sun}

Tianjin University of Traditional Chinese Medicine

\section{Chunying Tian}

Tianjin University of Traditional Chinese Medicine

Zhikui Tian

Tianjin University of Traditional Chinese Medicine

\section{Huaien Bu}

Tianjin University of Traditional Chinese Medicine

Hongwu Wang

Tianjin University of Traditional Chinese Medicine

\section{Study protocol}

Keywords: Essential hypertension, Baduanjin, Physical fitness, Martial arts, Blood pressure

Posted Date: January 12th, 2021

DOI: https://doi.org/10.21203/rs.3.rs-63424/v1

License: (1) (i) This work is licensed under a Creative Commons Attribution 4.0 International License. Read Full License 


\section{Abstract}

Background: Clinical treatment of hypertension recommends the use of drugs to reduce the incidence and mortality of cardiovascular diseases in hypertensive patients. However, the side effects of long-term medication and economic pressure have increased the medical community's interest in Baduanjin as a method of exercise therapy. Clinical studies have shown that Baduanjin can be used to treat various diseases, and it can also regulate physical and mental factors such as tension and anxiety. This study aims to evaluate the antihypertensive effect of Baduanjin on patients with essential hypertension.

Methods/design: In this study, 92 patients with essential hypertension were randomly divided into the Baduanjin group and the routine care group, using a randomized, active control, evaluator blind method, and two parallel control methods, with 46 cases in each group, signed informed Subjects with the consent form will manage their blood pressure by limiting salt intake, losing weight, drinking moderately, exercising, and adjusting dietary intake during the first visit. In addition to self-management, the Baduanjin group will also conduct 30-minute Baduanjin training three times a week for a total of 8 weeks. The main result is the measurement of blood pressure. In addition, body composition will be measured as a secondary result.

Discussion: This study is a randomized controlled Baduanjin trial and is not currently widely used. It may provide valuable data on the impact of Baduanjin on essential hypertension, which will inform non-drug treatment options for this disease.

Trial registration: Chinese Clinical Trial Registration Center, ChiCTR200003570. Register on August 16, 2020.

\section{Background}

Hypertension is a clinical syndrome characterized by increased systemic arterial pressure. Essential hypertension is a major health problem facing the world, which can cause cardiovascular events and lead to higher mortality. The fast-paced contemporary life has changed people's dietary structure and living habits. Excessive sugar, highprotein and high-fat food intake, unhealthy work and rest habits, and insufficient exercise are causing more and more patients with hypertension. [1-2]. It poses a major threat to public health [3]. At present, a quarter of the world's population is threatened by hypertension. In developed countries, the prevalence rate of hypertension is $37.3 \%$, and in developing countries it is $22.9 \%$. It is estimated that by 2025 , the global proportion is expected to rise to $29 \%$ [4]. If the blood pressure is not controlled, blood vessel damage is prone to occur, leading to complications of hypertension [5]. This complication is very serious, such as myocardial infarction, stroke, and renal failure [6]. The onset of essential hypertension is hidden, the course lasts for a long time, and it often accompanies a lifetime [7]. This makes the daily care and health care of patients with essential hypertension particularly important [8].

The current goal of prevention and treatment of hypertension is to reduce the morbidity and mortality of cardiovascular diseases by maintaining normal blood pressure $[9,10]$. In the clinical treatment of SBP, it is generally recommended to take drugs for the treatment of hypertension [11]. However, taking into account the side effects and economic factors associated with long-term medication, non-drug treatment methods, such as healthy eating, exercise, smoking cessation, and moderate drinking, are widely used to treat and manage hypertension in addition to drug therapy [12]. The European Society of Hypertension (ESH) was the first to recommend that patients with essential hypertension should appropriately increase physical activity, increase muscle strength and 
balance. Studies have found that appropriate increase in physical exercise in patients with essential hypertension can effectively reduce the level of oxidative stress markers [13-15]. Baduanjin was used as a kind of exercise therapy in ancient China to treat various diseases. The interest in modern research is increasing day by day [16, 17]. According to a study by Chen [18], Baduanjin may decrease sympathetic tone and increase parasympathetic tone, which may cause changes in the autonomic nervous system. In addition, a systematic review of a randomized controlled study conducted by Guan et al. [19] found that a certain period of Baduanjin exercise can regulate blood lipids and improve cognitive function [20].

This article analyzes the blood pressure and body composition of patients with essential hypertension diagnosed by hospital examination. We will evaluate the effect of Baduanjin by comparing the control of routine care group and routine care plus Baduanjin experimental group on hypertension. Therefore, the effectiveness of Baduanjin in the treatment of essential hypertension will be evaluated in this randomized, active-controlled, evaluator-blinded, double-parallel controlled trial.

\section{Methods/design}

\section{Study design}

This is a double-blind, multi-center, in line with the comprehensive report review criteria for randomized controlled clinical trials (CONSORT 2010) guidelines (Fig. 1) [21]. We designed the trial based on the "Standard Protocol Project: Recommendations for Interventional Trials" (SPIRIT) statement (Table 1) and its corresponding checklist (Additional Document 1) [22]. It will be carried out in the Second Affiliated Hospital of Tianjin University of Traditional Chinese Medicine and Tianjin First Central Hospital. The research protocol was registered in the Chinese Clinical Trial Registry on August 16, 2020, and received the registration number (ChiCTR2000035710). 
Table 1

Schedule of enrollment, intervention, and assessments

\begin{tabular}{|c|c|c|c|c|c|c|c|c|c|c|c|c|c|c|}
\hline \multirow[b]{3}{*}{ TIMEPOINT** } & \multicolumn{14}{|c|}{ STUDY PERIOD } \\
\hline & \multirow{2}{*}{$\begin{array}{l}\text { Enrolment } \\
-t_{1}\end{array}$} & \multirow{2}{*}{$\begin{array}{l}\text { Allocation } \\
0\end{array}$} & \multicolumn{8}{|c|}{ Post-allocation } & \multicolumn{4}{|c|}{ Close-out } \\
\hline & & & $t_{1}$ & $t_{2}$ & $t_{3}$ & $t_{4}$ & $t_{5}$ & $t_{6}$ & $t_{7}$ & $t_{8}$ & $t_{9}$ & $t_{10}$ & $t_{11}$ & $t_{12}$ \\
\hline \multicolumn{15}{|l|}{ ENROLMENT: } \\
\hline Eligibility screen & $x$ & & & & & & & & & & & & & \\
\hline Informed consent & $x$ & & & & & & & & & & & & & \\
\hline Allocation & & $x$ & & & & & & & & & & & & \\
\hline \multicolumn{15}{|l|}{ INTERVENTIONS: } \\
\hline \multicolumn{15}{|l|}{ Treatment group } \\
\hline \multicolumn{15}{|l|}{ Control group } \\
\hline \multicolumn{15}{|l|}{ ASSESSMENTS: } \\
\hline Study agreement & $x$ & $x$ & & & & & & & & & & & & \\
\hline $\begin{array}{l}\text { Check for } \\
\text { participation in } \\
\text { other clinical trials }\end{array}$ & & $x$ & & & & & & & & & & & & \\
\hline $\begin{array}{l}\text { Sociodemographic } \\
\text { characteristics }^{1}\end{array}$ & & $x$ & & & & & & & & & & & & \\
\hline $\begin{array}{l}\text { Baduanjin } \\
\text { exercises }\end{array}$ & & & $x$ & $x$ & $x$ & $x$ & $x$ & $x$ & $x$ & $x$ & & & & \\
\hline $\begin{array}{l}\text { Body composition } \\
\text { test }\end{array}$ & & $x$ & $x$ & & & $x$ & & & & $x$ & & & & \\
\hline $\begin{array}{l}\text { Measurement of } \\
\text { blood pressure }\end{array}$ & & $x$ & $x$ & & & $x$ & & & & $x$ & & & & \\
\hline $\begin{array}{l}\text { Number of } \\
\text { Baduanjin } \\
\text { sessions } \\
\text { attended }\end{array}$ & & & & & & & & & & & $x$ & $x$ & $x$ & $x$ \\
\hline Adverse events & & & & & & $X$ & & & & $\mathbf{x}$ & 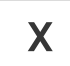 & $x$ & $x$ & $X$ \\
\hline
\end{tabular}

\section{Participants}

\section{Inclusion criteria}

To enroll in the trial, patients must meet all the inclusion criteria: 
(1) Patients diagnosed as essential hypertension by the hospital

(2) Between the ages of $18-65$

(3) Understand the research process in detail and be able to follow the suggestions provided by the researcher.

(4) Sign the research agreement and agree to participate in the research voluntarily.

\section{Exclusion criteria}

Subjects may not participate in the trial if they:

(1) Patients who are pregnant or breastfeeding

(2) Patients with hypertension are deemed to have limited joint mobility and cannot be treated with exercise.

(3) Inability to communicate normally, such as suffering from dementia or mild cognitive impairment.

(4) If they participated in another trial within one month before this study.

\section{Discontinuation and dropout criteria}

Subjects will be eliminated from the trial if:

(1) The patient was found to have a previously undiagnosed serious disease after registration and before the start of the clinical trial.

(2) The patient has other diseases (except hypertension) that may affect the test results.

(3) The patient cannot participate in the follow-up for any reason.

(4) The patient fails to comply with the treatment or fails to provide important information for the evaluation.

(5) The patient asked to withdraw from the study.

\section{Recruitment}

The recruitment of patients will be advertised in the bulletin board and outpatient office of the Second Affiliated Hospital of Tianjin University of Traditional Chinese Medicine and Tianjin First Central Hospital (TJFCH). Patients who intend to participate in the study will be screened first to determine whether they meet the inclusion criteria. Eligible patients will be fully explained by the study and can voluntarily decide whether they are willing to participate. If the patient agrees to the study terms, our professional researchers will check whether they meet the inclusion and exclusion criteria. Then, the patients were randomly divided into the Baduanjin group or the routine care group. After the task is completed, the researchers will arrange their treatment procedures. The first participant to register on August 18, 2020.

\section{Randomization}

Statisticians who are not involved in conducting research or evaluating clinical trials use the statistical program SAS to generate random sequences. The statistician will use the sequence to prepare 92 sealed envelopes, 46 of which will contain 46A and 46B based on closed randomization. After the participant has signed the informed 
consent form, the researcher will open the next envelope in the sequence, and Participants' information was notified to the experimental group or the routine care group (Fig. 1).

\section{Blinding}

Since blinding of the intervention is impossible, so group assignments will not be hidden and participants will not be blind. But what we can do is that the outcome assessors and statistical analysts will be blind to the allocation, and they will not participate in the patient recruitment or intervention process. The outcome assessor will assess the participants included in the study. Statistical analysts will analyze statistical data to prevent selective reporting bias. Blindness is only allowed to be relieved if there are serious side effects when the intervention measures assigned by the participant are disclosed (the specific situation is at the discretion of the evaluator).

\section{Education levels of the practitioners}

All researchers participating in the study have a medical license issued by the Ministry of Health and have at least one year of medical clinical experience. These doctors will be fully trained so that they strictly abide by the research protocol and are familiar with research treatment methods. All Baduanjin educators have received training from professional rehabilitation doctors or Baduanjin sports experts, and have at least one year of Baduanjin sports experience.

\section{Intervention}

At the beginning of the study, both groups of patients will receive education to limit salt intake, moderate diet, moderate drinking, exercise, and a reasonable and healthy diet to manage their blood pressure. We also specifically ask the two groups of patients not to do any strenuous exercise that may affect the test results. After the education is over, the routine care group will conduct self blood pressure management for 4 weeks.

\section{Treatment group}

On the basis of self-management, this group implemented Baduanjin as a learning intervention. In this study, the "Ba Duan Jin" published by the State Sports General Administration was performed 3 times a week for 30 minutes each time for 8 weeks. The whole set of Baduanjin exercises includes eight postures (Fig. 2). In each course of treatment, in order to maximize compliance with the research protocol, the intervention will be mainly carried out in the outpatient ward of TJFCH. If group meetings are required, practitioners can visit external locations. Patients in the experimental group will receive a reference book about Baduanjin, which can promote the accuracy and effectiveness of their practice. Everyone will record the number of exercises and time per day.

\section{Control group}

During the 8-week intervention period, patients in the control group will not receive any specific exercise training. In the 4th and 8th weeks, both groups will go to the hospital for evaluation. After the treatment, we will follow up the two groups of patients for 4 weeks.

\section{Outcome assessment}

At the beginning of the study, researchers will explain the study plan to patients during the screening process. In addition, the patients included in the study were asked about their sociodemographic characteristics, including age, gender, occupation, past medical history, current disease and type of oral medication. During the research 
process, if adverse events occur, they will be recorded. Researchers will determine the severity of the adverse events and decide whether to continue the trial. Follow-up evaluation will be performed 4 weeks after the first screening (Table 1, Fig. 1).

\section{Primary outcome}

This test uses blood pressure (BP) as the main monitoring result. The researchers will use an automatic electronic blood pressure monitor (HEM-7211, Omron Health Medical Co., Ltd., China) to assess the patient's BP in a steady state. In order to achieve the accuracy of the data obtained, the researcher will take three measurements of the patient's BP and calculate the average of the three measurements as the result. BP will be measured at baseline (assessment 1), before each visit (assessment 2 and 3), and during follow-up (assessment 4). The primary endpoint is week 8 (assessment 3).

\section{Secondary outcome measurements}

The secondary result of this test is the patient's body composition. Researchers will use the BCA-2A body composition analyzer (BCA-2A; Tongfang Health Technology Co., Ltd., China) to test and evaluate the weight, body mass index, basal metabolic rate, muscle mass, total water, and intracellular water of each patient., Extracellular water, protein, bone mass, fat mass, skeletal muscle, BMI( Body mass index), WHR(Waist and hip ratio), body age, health score, visceral fat mass. The patient will stand barefoot on the BCA-2A body composition analyzer. After measuring the weight first, the patient holds two handles with both hands, and the machine will transmit signals of multiple frequencies through their bodies to obtain the impedance corresponding to each frequency. Using these measured impedance values, the machine will calculate how much body fat and water they have. Testing will be performed at baseline (assessment 1), week 8 (assessment 3) and follow-up (assessment 4).

\section{Sample size}

The following formula was used to estimate the required sample size:

$$
n=\frac{\left(Z_{1-\alpha / 2}+Z_{1-\beta}\right)^{2} \times\left(\sigma_{1}^{2}+\sigma_{2}^{2}\right)}{\delta^{2}}
$$

In the formula, $\mathrm{n}$ is the sample content of each group, $\sigma$ is the standard deviation, and the clinically significant difference between the two groups is represented by $\delta$. Using the parameters $a=0.05$ and POWER $=80 \%$, the $Z$ value is defined as $Z_{1-\alpha / 2}=1.96, Z_{1-\beta}=0.84$. Considering the loss rate is $15 \%$, the final number of samples in each group is 46 , so the total number of samples in this study is 92 .

\section{Statistical analysis plan}

Sample distribution: The overall quantity of each data set will be described, and if there is any missing, it will be described in detail. Balanced comparison: The baseline data of the data set will be compared to assess the comparability of the data between the two groups. Efficacy analysis: In this study, the difference in patient blood pressure changes was used as the main statistical analysis, and the effectiveness of Baduanjin will be tested by calculating the difference between maximum systolic blood pressure and minimum diastolic blood pressure before (baseline) and after treatment (week 8) for each subject. The comparison of intra-group differences will use paired $\mathrm{t}$ - test, and the comparison of inter-group differences will use independent $\mathrm{t}$ - test. the nonparametric test 
(Wilcoxon symbolic rank sum test or Wilcoxon rank sum test) is used if the data does not conform to the normal distribution.

Body composition analysis will be analyzed through the above analysis plan. Information about adverse events will be collected through patient reports and observations by researchers. According to the definition of adverse events, specific adverse events will be listed, as well as their severity, causes and explanations. The number of adverse events and the incidence of adverse events will be described statistically and will be analyzed by chisquare test or Fisher's exact test.

The statistical test will be two-tailed, and a $\mathrm{P}$ value of $<0.05$ is considered significant. The data will be processed and analyzed using the principle of intention to treat. The last observation, carry-over, and multiple imputation that are widely used in clinical research will be used for missing data, and multiple imputation or regression analysis will be used to check whether there are any differences. All statistical calculations will use SPSS19.0 software.

\section{Patient safety}

The intervention method used in this study is a simple exercise, not an unapproved drug or medical device, and the occurrence of adverse events is minimal. However, the researchers will inform the patients of all possible adverse events after the Baduanjin exercise, and inform the patients to report and record any such adverse events in time. This research will be regularly monitored by the China Clinical Trial Monitoring Committee. The data management committee will be composed of a statistician and a clinical research methodology expert to plan the research progress, check whether the research has followed the research plan, and consult all case report forms. During the trial period, no audits are planned, and no mid-term analysis is planned.

\section{Ethics and dissemination}

This research complies with the principles of the "Declaration of Helsinki" and related ethical guidelines concerning informed consent, confidentiality and data storage. Clinical researchers will follow the research plan and will actively respond to any questions raised by participating patients. Additional file 1 contains the complete SPIRE checklist.

\section{Trial status}

The current agreement is version 1.5, and recruitment will begin on August 31, 2020. The completion date of the recruitment is September 2020. The trial was per-registered before the start of recruitment.

\section{Discussion}

As a kind of traditional Chinese medicine qigong, Baduanjin usually involves exercises that integrate the body and mind, which can balance the body's yin and yang, promote blood circulation, and maximize physical and mental health [23]. More and more studies have found that combining with Baduanjin exercise can significantly improve the prognosis of clinical chronic diseases [24]. In the study of Ye et al., it was found that Baduanjin exercise improves the balance and quality of life of the elderly with chronic diseases [25], and that Baduanjin promotes the rehabilitation of patients with cardiovascular diseases [26]. It is worth noting that these studies on Baduanjin are mainly elderly participants or the recovery period of cardiovascular events caused by hypertension.

This study has certain potential limitations as an interventional study. Participants in this study cannot be blind. As an experimental group, you need to practice Baduanjin, so patients will know whether they belong to the 
experimental group or the control group. This factor may influence other behaviors to influence the results of the study. However, the Baduanjin sports coach will not participate in the recruitment, evaluation and data analysis process of this study, which will minimize the deviation. The advantage of this study is that it will evaluate adults with essential hypertension between the ages of 18 and 65, not just the elderly. In addition, Baduanjin exercises are gentle and easy to master. Almost anyone can practice at home, reducing social medical expenses. Therefore, this study may provide valuable data for the influence of Baduanjin on essential hypertension.

\section{Abbreviations}

ESH

European Society of Hypertension

CONSORT

criteria for randomized controlled clinical trials

SPIRIT

Standard Protocol Project:Recommendations for Interventional Trials

TJFCH

Tianjin First Central Hospital

BP

Blood pressure

\section{Declarations}

\section{Acknowledgements}

The author thanks all participants.

\section{Authors' contributions}

YYG designed the research and drafted the manuscript. YH, HEB plan statistical strategy. DJW and ZKT actively participate in sample size calculation and random allocation. HWW participated in the research design. YG, CYT, XS monitor communications during the trial. All authors have read, revised, and approved the final version of the manuscript.

\section{Funding}

This research was supported by the National Key Research and Development Program of China (approval No.: 2017YFC1703305) and the Basic Research on Health Identification of Traditional Chinese Medicine in Tianjin Colleges and Universities "Innovation Team Training Plan" (approval No.: TD13- 5049). The funders decide to submit the report for publication in the research design, collection, management, report writing or data analysis or interpretation of the report; they also do not have the ultimate authority in any of these activities.

\section{Availability of data and materials}

The data sets used or analyzed during the study can be requested from the corresponding author.

\section{Ethics approval and consent to participate}


The research protocol has passed the ethics committee of Tianjin University of Traditional Chinese Medicine (approval number: TJUTCM-EC20190004). Before all participants participate in the trial, a signed consent form will be obtained from them.

\section{Consent for publication}

Not applicable.

\section{Competing interests}

The authors declare that they have no competing interests.

\section{References}

1. Zhang X, Huang L, Peng X, Xie Y, Bao X, Huang J, et al. Association of handgrip strength with hypertension among middle-aged and elderly people in Southern China: A cross-sectional study. Clinical and experimental hypertension (New York, NY: 1993). 2020;42(2):190-6.

2. Mancia G, Fagard R, Narkiewicz K, Redon J, Zanchetti A, Böhm M. et al. 2013 ESH/ESC guidelines for the management of arterial hypertension: the Task Force for the Management of Arterial Hypertension of the European Society of Hypertension (ESH) and of the European Society of Cardiology (ESC). European heart journal. 2013;34(28):2159 - 219.

3. Kearney PM, Whelton M, Reynolds K, Muntner P, Whelton PK, He J. Global burden of hypertension: analysis of worldwide data. Lancet. 2005;365(9455):217 - 23.

4. Mills KT, Bundy JD, Kelly TN, Reed JE, Kearney PM, Reynolds K. et al. Global Disparities of Hypertension Prevalence and Control A Systematic Analysis of Population-Based Studies From 90 Countries. Circulation. 2016;134(6):441-+.

5. Yannoutsos A, Mourad JJ, Blacher J, Safar M. [Hypertension and cardiovascular risk: the J-curve concept]. Praxis. 2010;99(22):1335-41.

6. Zhou X, Frohlich ED. Analogy of cardiac and renal complications in essential hypertension and aged SHR or L-NAME/SHR. Medicinal chemistry (Shariqah (United Arab Emirates)). 2007;3(1):61 - 5.

7. Keith NM, Wagener HP, Barker NW. Some different types of essential hypertension: their course and prognosis. Am J Med Sci. 1974;268(6):336 - 45.

8. Verdecchia P, Angeli F, Gattobigio R, Porcellati C. Ambulatory blood pressure monitoring and prognosis in the management of essential hypertension. Expert Rev Cardiovasc Ther. 2003;1(1):79-89.

9. Carlberg B, Nilsson PM. Hypertension in the elderly: what is the goal blood pressure target and how can this be attained? Curr Hypertens Rep. 2010;12(5):331-4.

10. Mayer M. Qigong and hypertension: a critique of research. Journal of alternative and complementary medicine (New York, NY). 1999;5(4):371 - 82.

11. Bakris G, Ali W, Parati G. ACC/AHA Versus ESC/ESH on Hypertension Guidelines: JACC Guideline Comparison. J Am Coll Cardiol. 2019;73(23):3018-26.

12. Lopes S, Mesquita-Bastos J, Alves AJ, Ribeiro F. Exercise as a tool for hypertension and resistant hypertension management: current insights. Integrated blood pressure control. 2018;11:65-71. 
13. Yu Y, Gao Q, Xia W, Zhang L, Hu Z, Wu X. et al. Association between Physical Exercise and Biomarkers of Oxidative Stress among Middle-Aged and Elderly Community Residents with Essential Hypertension in China. BioMed research international. 2018;2018:4135104.

14. Korsager Larsen M, Matchkov VV. Hypertension and physical exercise: The role of oxidative stress. Medicina. 2016;52(1):19-27.

15. Roque FR, Briones AM, García-Redondo AB, Galán M, Martínez-Revelles S, Avendaño MS. et al. Aerobic exercise reduces oxidative stress and improves vascular changes of small mesenteric and coronary arteries in hypertension. Br J Pharmacol. 2013;168(3):686-703.

16. Tao J, Liu J, Chen X, Xia R, Li M, Huang M. et al. Mind-body exercise improves cognitive function and modulates the function and structure of the hippocampus and anterior cingulate cortex in patients with mild cognitive impairment. Neurolmage Clinical. 2019;23:101834.

17. Xie Y, Guo F, Lu Y, Guo Y, Wei G, Lu L. et al. A 12-week Baduanjin Qigong exercise improves symptoms of ankylosing spondylitis: A randomized controlled trial. Complement Ther Clin Pract. 2019;36:113-9.

18. Chen TT, Yue GH, Tian YX, Jiang CH. Baduanjin Mind-Body Intervention Improves the Executive Control Function. Front Psychol. 2017;7:8.

19. Mei L, Chen Q, Ge L, Zheng G, Chen J. Systematic review of chinese traditional exercise baduanjin modulating the blood lipid metabolism. Evidence-based complementary alternative medicine: eCAM. 2012;2012:282131.

20. Tao J, Chen X, Egorova N, Liu J, Xue X, Wang Q. et al. Tai Chi Chuan and Baduanjin practice modulates functional connectivity of the cognitive control network in older adults. Scientific reports. 2017;7:41581.

21. Schulz KF, Altman DG, Moher D. CONSORT 2010 statement: updated guidelines for reporting parallel group randomised trials. BMJ. 2010;340:c332.

22. Chan AW, Tetzlaff JM, Altman DG, Laupacis A, Gotzsche PC, Krleza-Jeric K. et al. SPIRIT 2013 Statement: Defining Standard Protocol Items for Clinical Trials. Ann Intern Med. 2013;158(3):200-+.

23. Ho RTH, Wan AHY, Chan JSM, Ng SM, Chung KF, Chan CLW. Study protocol on comparative effectiveness of mindfulness meditation and qigong on psychophysiological outcomes for patients with colorectal cancer: a randomized controlled trial. BMC Complement Altern Med. 2017;17(1):390.

24. Xiao CM, Zhuang YC. Effect of health Baduanjin Qigong for mild to moderate Parkinson's disease. Geriatr Gerontol Int. 2016;16(8):911-9.

25. Ye J, Simpson MW, Liu Y, Lin W, Zhong W, Cai S. et al. The Effects of Baduanjin Qigong on Postural Stability, Proprioception, and Symptoms of Patients With Knee Osteoarthritis: A Randomized Controlled Trial. Frontiers in medicine. 2019;6:307.

26. Mao S, Zhang X, Chen M, Wang C, Chen Q, Guo L, et al. Beneficial Effects of Baduanjin Exercise on Left Ventricular Remodelling in Patients after Acute Myocardial Infarction: an Exploratory Clinical Trial and Proteomic Analysis. Cardiovascular drugs and therapy. 2020.

\section{Figures}




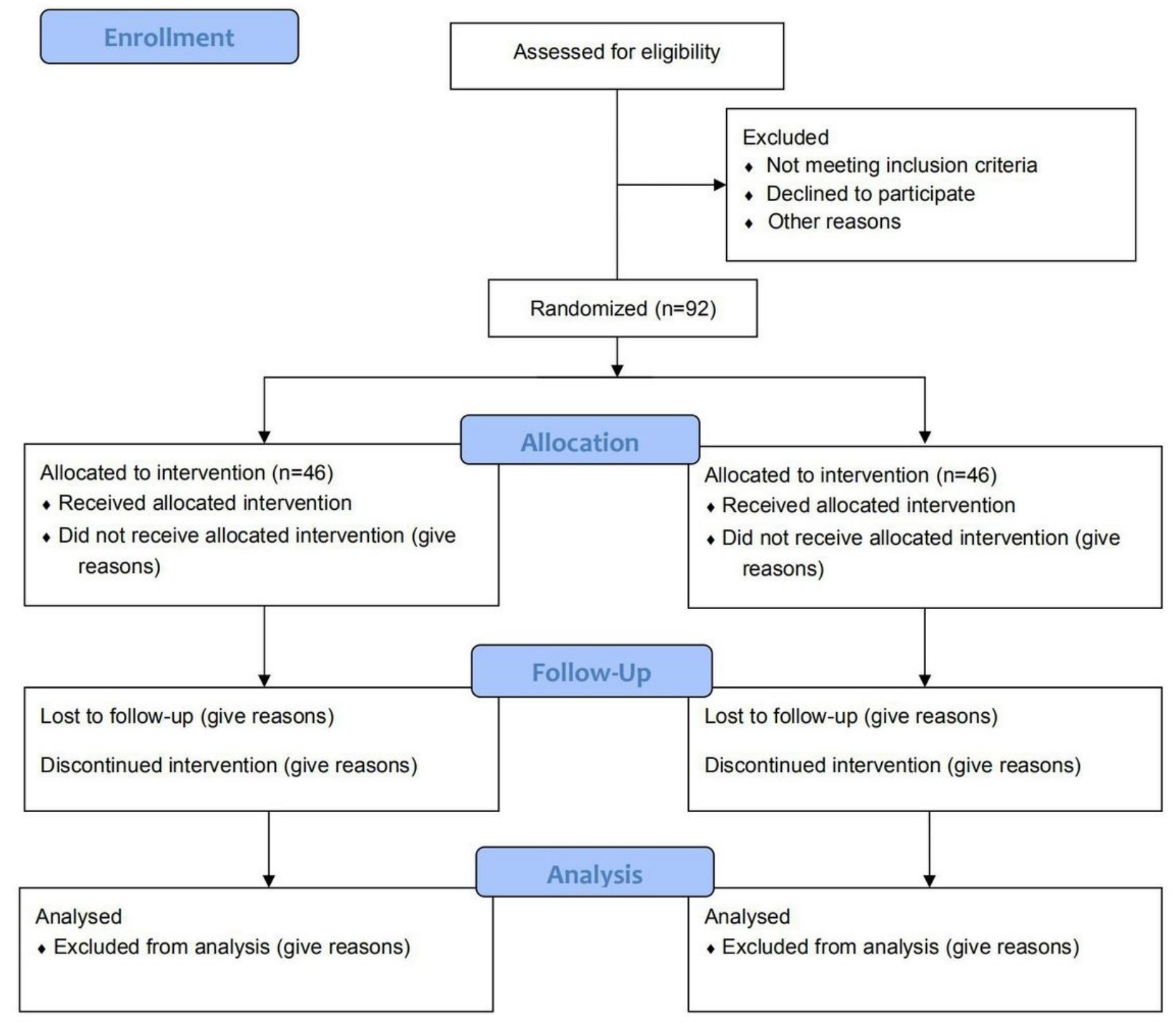

Figure 1

Flowchart outlining the study timeline, including enrollment, allocation, follow-up, and analysis

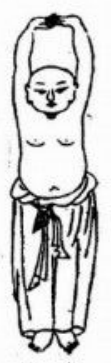

(1)

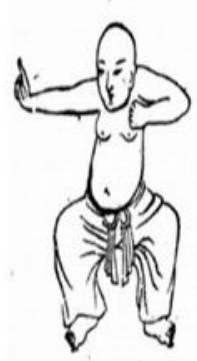

(2)

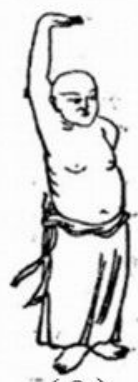

(3)

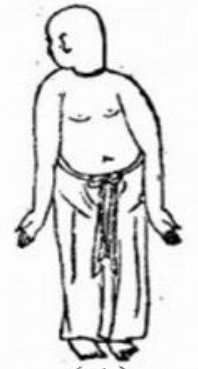

(4)

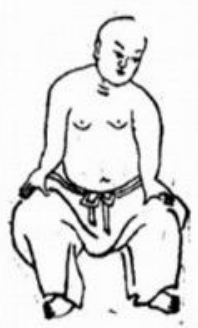

(5)

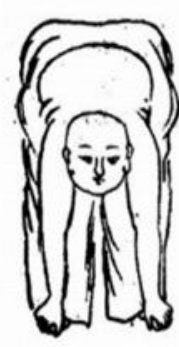

(6)

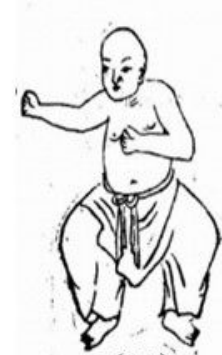

$(7)^{-1}$

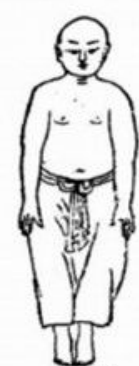

(8) 
Figure 2

Diagram of The Baduanjin exercise.

\section{Supplementary Files}

This is a list of supplementary files associated with this preprint. Click to download.

- SPIRITChecklistdownload8Jan13.doc 